\title{
Vitamin D supplementation in inflammatory bowel disease: the role of dosage and patient compliance
}

\author{
Kojecky $\mathrm{V}^{1}$, Adamikova $\mathrm{A}^{1}$, Klimek $\mathrm{P}^{2}$ \\ Internal Clinic, Bata Regional Hospital, Zlin, Czech Republic. kojecky@bnzlin.cz
}

\begin{abstract}
OBJECTIVES: Vitamin D substitution is recommended in patients with inflammatory bowel disease. Specific guidelines are lacking. The aim of this study was to assess the effect of vitamin $D$ supplementation with respect to dosage and patient compliance.

METHODS: A prospective cohort study of $167 \mathrm{Crohn}$ disease/ulcerative colitis outpatients. Patients were screened for serum vitamin $\mathrm{D}\left(25 \mathrm{OHD}_{2+3}\right)$ at the end of summer and in late winter. Demographic data, history of vitamin $\mathrm{D}$ supplementation were recorded and matched with prescription records.

RESULTS: A total of 57 subjects used vitamin D supplementation (mean dose $1104 \mathrm{IU} /$ day). $25 \mathrm{OHD}_{2+3}$ levels were lower $(p<0.001)$ in winter both in substituted and unsubstituted group, without any differences between groups within the same season. $250 \mathrm{HD}_{2+3}$ levels did not correlate with the substitution dose. $52.1 \%$ of subjects were fully compliant with substitution. $25 \mathrm{OHD}_{2+3}$ and prevalence of vitamin $\mathrm{D}$ deficit in this group were comparable with unsubstituted subjects except a higher prevalence of vitamin $D$ insufficiency $(p<0.02)$.

CONCLUSION: Fixed dosage of 1100 IU/day of vitamin D was insufficient to correct the deficiency. Patient compliance with vitamin $D$ supplementation was low, however this fact did not significantly contribute to the degree of vitamin D deficiency in this dosage (Tab. 3, Fig. 1, Ref. 21). Text in PDF www.elis.sk.

KEY WORDS: inflammatory bowel disease, vitamin D, supplementation, Crohn's disease.
\end{abstract}

Abbreviations: IBD-inflammatory bowel disease, vitD-vitamin D, UC - ulcerative colitis, CD - Crohn's disease, RCT - randomized controlled trial.

\section{Introduction}

Inflammatory bowel disease (IBD) belongs to the group of diseases caused by abnormal function of the immune system in genetically susceptible subjects, whereby environmental factors play a role. Vitamin D (vitD) is traditionally regarded as the major regulator of calcium and bone metabolism. VitD receptors are present in most tissues of the body including the immune system. VitD appears to have an important role in the regulation of innate and adaptive immunity. An increasing body of literature supports the important role of vitD in the development and course of IBD.

VitD deficiency is common both in general population and in the IBD patients with significant seasonal variation (1). A number of other factors associated with low vitD levels was identified in the IBD subjects, e.g. smoking, small bowel resection, a longstanding disease.

${ }^{1}$ Internal Clinic, Bata Regional Hospital, Zlin, and ${ }^{2}$ Department of Statistics and Quantitative Methods, Faculty of Management and Economics, Tomas Bata University in Zlín, Czech Republic

Address for correspondence: V. Kojecky, Internal Clinic, Bata Regional Hospital Zlin, Havl. nab. 600, CZ- 76001 Zlin, Czech Republic Phone: +420577552512, Fax: +420577552755
Data supporting clinical association between vitD levels and the course of the disease are conflicting. However, there are human studies demonstrating that vitD supplementation may favourably modify the severity of the disease (2).

Recommended daily dietary intake of vitD for adult population ranges from $600 \mathrm{IU}$ to $800 \mathrm{IU}$ per day. Any references for the IBD patients are missing. It has been hypothesised that optimal supplementation doses could be higher than those for general population. This opinion is based on data coming from the controlled studies with patient compliance monitored (3). An exact impact of vitD supplementation on the said deficiency in general clinical practice, where other factors like patient compliance play a role, is almost unknown.

The aim of this prospective study was to assess the efficiency of vitD supplementation with respect to dosage and patient compliance, as well as factors that may affects vitD levels (disease type, activity, ileal resection, steroid treatment).

\section{Materials and methods}

This was a prospective cohort study of the IBD (Crohn's disease/ulcerative colitis) outpatients from a tertiary IBD center and covering the period from January 2013 to April 2015.

A total of 167 patients aged 18 or older with confirmed diagnosis of IBD (based on endoscopic, histologic or radiologic findings) were enrolled. The patients were considered taking vitamin D supplements if they were taking any preparation containing vitD 
at any time during the 3 months before the vitD assessment. Those taking no vitD were assigned as a control group.

Exclusion criteria included conditions that may affect serum vitD levels (renal insufficiency, liver and cholestatic disease, malabsorption, coeliac disease, treatment with anticonvulsants, pregnancy, gastrointestinal surgery and/or any other severe disease) or impossibility to obtain valid data $(4,5,6,7)$. Demographic data, medical history and medication were obtained from our medical database and a questionnaire completed at each subject's visit. The patients were asked to report their oral vitD supplementation (including daily/weekly dosages and type of supplementation including over-the-counter supplements), and data were matched with previous prescription records. The disease activity for ulcerative colitis (UC) was determined using a partial Mayo score, where the score of $\geq 3$ was considered as a clinically active disease. In case of Crohn's disease (CD), the activity was assessed using the Crohn Disease Activity Index (CDAI), where clinically active disease was defined by a score $\geq 150$.

At patient visit, blood samples were taken to evaluate CRP, alkaline phosphatase (ALP) and serum vitD concentration. The blood samples were obtained at the end of summer (SeptemberDecember) and in late winter (January-April), with a minimum 4-month delay between samples in each subject.

VitD levels $\left(25 \mathrm{OHD}_{2}+25 \mathrm{OHD}_{3}\right)$ were determined using an immunochemiluminescent assay (Abbotts Architect, cut off $>75$ $\mathrm{nmol} / \mathrm{l})$. Vitamin D deficit was defined as $25 \mathrm{OHD}_{2+3}<50 \mathrm{nmol} / \mathrm{L}$, insufficiency as $25 \mathrm{OHD}_{2+3}$ between 50 and $74 \mathrm{nmol} / \mathrm{L}$, while levels between 75 and $250 \mathrm{nmol} / \mathrm{L}$ were considered normal (8).
A written informed consent was obtained from each patient, and the study was approved by the local ethical committee.

A statistical analysis was performed using XLStatistics and Minitab 14. Univariate statistical analyses were performed using tests (unpaired T test, Mann-Whitney test) based on a data normality check. Categorical determinants were analysed using Pearson's Chi-square test (or Fisher's exact test, whenever anticipated frequencies were low). Regression analyses were performed using Pearson and/or Spearman's correlation coefficients. $p$ values $<0.05$ were considered as significant.

\section{Results}

A total of 167 IBD subjects were included in this study. 57 patients reported vitD supplementation. There were no significant differences in baseline characteristics between the groups with and without supplementation except a higher proportion of female subjects in the group with vitD supplementation (31.8 vs $54.4 \%$ ) (Tab. 1).

VitD levels were significantly lower in the late winter compared to the end of summer both in the supplemented $(p=0.007)$ and non-supplemented groups $(\mathrm{p}<0.001)$. The proportion of subjects with vitD deficit changed accordingly. It decreased significantly from winter to late summer (unsupplemented $52.7 \%$ vs $19.1 \%$, supplemented $40.4 \%$ vs $10.5 \%, \mathrm{p}<0.001$ ), and an inverse trend was apparent in the number of subjects with normal vitD levels (unsupplemented $15.6 \%$ vs $44.5 \%$, p < 0.001 , supplemented $9.5 \%$ vs $31.6 \%, \mathrm{p}=0.008)$. There was no difference in

Tab. 1. Baseline characteristics.

\begin{tabular}{|c|c|c|c|}
\hline & $\begin{array}{l}\text { No substitution } \\
\mathrm{n}=110\end{array}$ & $\begin{array}{l}\text { vitD substitution } \\
\qquad \mathrm{n}=57\end{array}$ & $\mathrm{p}$ \\
\hline Age $(\mathrm{yr})$, mean $\pm \mathrm{SD}$ & $40.1 \pm 13.4$ & $36.3 \pm 10.0$ & NS* \\
\hline Female, n $(\%)$ & $35(31.8)$ & $31(54.4)$ & 0.007 \\
\hline Ulcerative colitis/Crohn disease, $\mathrm{n}(\%)$ & $34(30.9) / 76(69.1)$ & $21(36.8) / 36(63.2)$ & NS* \\
\hline Osteoporosis, $\mathrm{n}(\%)$ & $12(10.9)$ & $12(21.1)$ & NS* \\
\hline Ileal resection, n (\%) & $20(18.2)$ & $13(22.8)$ & NS* \\
\hline Disease duration (yr), mean \pm SD & $8.9 \pm 6.8$ & $7.1 \pm 6.0$ & NS* \\
\hline Active disease, $\mathrm{n}(\%)$ late summer & $17(15.5)$ & $9(15.8)$ & NS* \\
\hline Steroids, n (\%) & $14(12.7)$ & $13(22.8)$ & NS* \\
\hline Prednisone dose, mean \pm SD & $11.8 \pm 6.4$ & $6.5 \pm 4.5$ & 0.007 \\
\hline
\end{tabular}

* NS - not significant

Tab. 2. D vitamin levels in groups with and without supplementation.

\begin{tabular}{|c|c|c|c|}
\hline & $\begin{array}{l}\text { No supplementation } \\
\mathrm{n}=110\end{array}$ & $\begin{array}{l}\text { vitD supplementation } \\
\qquad \mathrm{n}=57\end{array}$ & $\mathrm{p}$ \\
\hline \multicolumn{4}{|l|}{ Late winter } \\
\hline $\mathrm{D}$ vitamin $(\mathrm{nmol} / \mathrm{L})$, mean $\pm \mathrm{SD}$ & $54.3 \pm 18.9$ & $56.3 \pm 22.2$ & NS* \\
\hline $\mathrm{D}$ vitamin deficit, $\mathrm{n}(\%)$ & $58(52.7)$ & $23(40.4)$ & NS* \\
\hline D vitamin insufficiency, $\mathrm{n}(\%)$ & $35(32.7)$ & $29(50.1)$ & 0.02 \\
\hline D vitamin normal, $\mathrm{n}(\%)$ & $17(15.6)$ & $5(9.5)$ & NS* \\
\hline \multicolumn{4}{|l|}{ Late summer } \\
\hline $\mathrm{D}$ vitamin $(\mathrm{nmol} / \mathrm{L})$, mean $\pm \mathrm{SD}$ & $70.7 \pm 22.2$ & $70.6 \pm 20.7$ & NS* \\
\hline D vitamin deficit, $\mathrm{n}(\%)$ & $21(19.1)$ & $6(10.5)$ & NS* \\
\hline D vitamin insufficiency, n (\%) & $40(36.4)$ & $33(57.9)$ & 0.009 \\
\hline D vitamin normal, $\mathrm{n}(\%)$ & $49(44.5)$ & $17(31.6)$ & NS* \\
\hline
\end{tabular}

* NS - not significant 
Tab. 3. Vitamin D supplementation dose.

\begin{tabular}{lcr}
\hline & \multicolumn{2}{c}{ Supplementation dose } \\
\cline { 2 - 3 } & recommended value & $1107 \pm 758$ \\
Daily supplementation dose, late winter (IU), mean \pm SD & $1160 \pm 690$ & $1100 \pm 892$ \\
Daily supplementation dose, late summer (IU), mean \pm SD & $1332 \pm 868$ & NS* \\
Cumulative dose in last 12 months (IU), mean \pm SD & & NS* \\
\hline
\end{tabular}

$\mathrm{IU}$ - international unit, * NS - not significant

the number of subjects with similar vitD status (except vitD insufficiency in the supplemented group) between both groups from winter to summer (Tab. 2).

No significant correlation was observed between vitD levels and other variables (sex, disease duration, CRP, ALP levels, presence of ileal resection, osteoporosis, disease activity, ulcerative colitis). Due to a small number of steroid-treated patients this subanalysis was not performed.

Recommended mean supplementation doses varied from 1160 to 1332 international units (IU)/day, and were stable during the year. Real doses as was reported by the subjects, were not significantly lower (1107 and 1100 IU/day). VitD levels did not correlate with the present real substitution dose (114-3000 IU/day) or with the cumulative dose within last 12 month (Tab. 3).

According to relevant medical records, overall 73 subjects should take vitD. However, vitD was actually used by 57 subjects only (78.1\%), and 19 of them did not follow recommended dosages (they used lower doses or omitted vitD irregularly). Only one half $(52.1 \%)$ of all subjects (38 out of 73 ) were fully compliant.

Subanalysis performed in the compliant subject group demonstrated that there was only a tendency to higher prevalence of vitD insufficiency compared to the unsupplemented group (winter $52.7 \%$ vs $32.7 \%$, p = 0.03, summer $57.9 \%$ vs $36.4 \%$, p = 0.02 ) (Fig. 1). The validity of these figures is limited due to a low number of subjects.

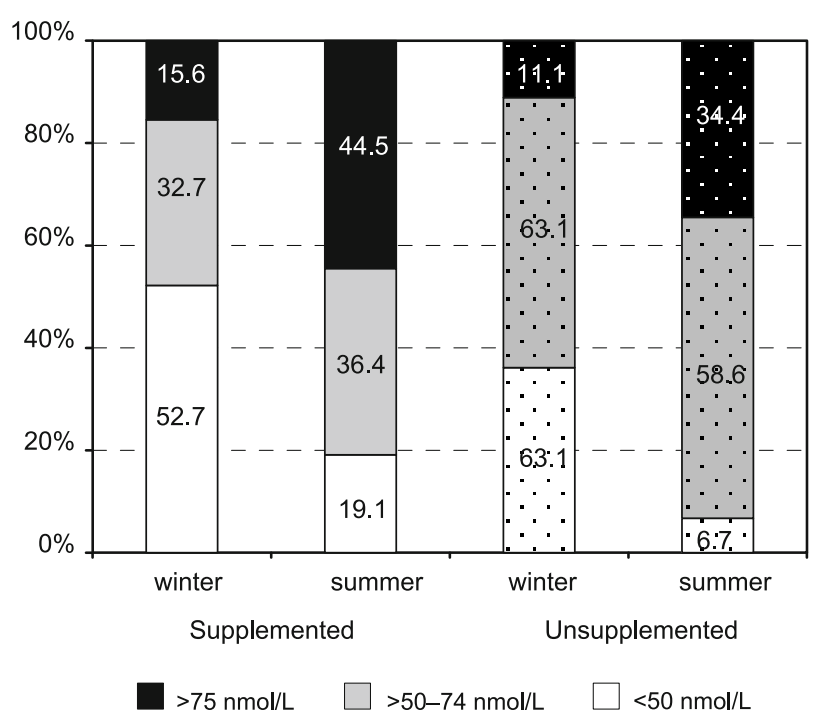

Fig. 1. Incidence of different degrees of vitamin $D$ deficiency in fully compliant subjects and in unsubstituted. Frequencies are shown in percentages. Vitamin D insufficiency winter $p=0.03$, summer $p=0.02$.

\section{Discussion}

This prospective cohort study demonstrated that various degrees of vitamin D deficiency were common in IBD patients. Our results are in line with other authors reporting low levels of vitD, with prevalence ranging widely from $18 \%$ to $95 \%(8,9)$. VitD deficit is frequent not only in the IBD patients, but in healthy population as well. In neighbouring Germany, $57 \%$ of healthy men and $58 \%$ of women had vitamin D levels below $50 \mathrm{nmol} / \mathrm{l}(10)$. Many risk factors for vitamin D deficiency were reported. Inadequate exposure to sunlight belongs to the most significant causes in general population (1) followed by an inadequate dietary intake. Other specific causes might be involved in the IBD patients, such as: impaired vitD absorption, intestinal resection, smoking, exposure to steroids, disease activity or genetic factors $(8,11,12,13)$. Their actual contribution to the deficit is unclear and the data are contradictory. The correlation between vitD levels and IBD activity was not clearly confirmed. Some authors, for example, found an inverse relation between vitD levels and the duration of disease activity (3), others confirmed this relation for Crohn's disease only, and not for ulcerative colitis (8, 13). Exploration of these relationships was not a primary goal of the present study; however, the authors were not able to confirm any link between vitD levels and other factors, such as gender, activity, type of IBD, disease duration, ileal resection, CRP, ALP levels. There is a general consensus that vitD deficiency has a negative impact on the state of health.

IBD patients with vitD deficiency tend to have a more aggressive course of the disease and require surgical management more frequently (14).

Recommendations for vitD substitution in IBD patients are not available. The ECCO Statement $13 \mathrm{C}$ suggests vitD supplementation for patients on steroid therapy and/or those with reduced bone density (doses 800-1000 IU/d) (15). Any guidelines for other patients or those in remission are lacking. Optimal supplementation dosage and regimen is not established, and it is not known, whether the dose may differ from otherwise healthy subjects. Present available data show that oral supplementation of 334-800 IU/ day decreased the risk of vitD deficiency; however, without any significant changes in plasma levels of vitD $(13,16)$. Higher doses of vitD (1200 IU daily) increased serum vitD levels from the mean $69 \mathrm{nmol} / \mathrm{L}$ to $96 \mathrm{nmol} / \mathrm{L} \mathrm{(3)}$. The present authors were not able to confirm this result. Comparable doses were used without affecting serum levels in accordance with Bour's study. The effect of our supplementation was minor and only apparent in a proportion of subjects with different degrees of vitD deficiency. The status shifted from a severe deficit to insufficiency in a larger number of supplemented patients compared to unsupplemented. 
One of the possible causes of vitD deficiency is an impaired vitD bioavailability. Ileal resection may interrupt enterohepatic circulation of bile acids and cause vitD malabsorption (17). A possible causal link between vitD deficit and ileal resection or CD localization was not confirmed by all. We did not note any dependency in our group. There are only few papers in literature, where vitD bioavailability after oral administration was studied. In one of them, Pappa et al (18) did not observe any difference in healthy subjects. VitD absorption varies significantly without any known underlying factor (19). Surprisingly, the said papers did not study patient compliance to supplementation, which may be lower in real life than in an RCT. Medication compliance is generally poor in IBD patients (30-70\%), as was evidenced for example in mesalazine or thiopurine treatments (20). Our group was not different. Out of the total number of subjects, in whom the physician felt they were taking the prescribed vitamin D, fully complied was only one half.

VitD levels between supplemented and unsupplemented subjects were comparable. These might represent another underlying factor. A shorter interval between sample collections were not considered to be important. A small number of subjects studied might have played a role along with the fact that supplementation was administered in recommended fixed doses which may have been insufficient.

As long as supplementation doses (1000 IU/D up to 5000 IU/D) were adjusted according to vitD levels, serum vitD rose effectively within 24 weeks (2). Pappa et al. used a different supplementation regimen and calculated the slope of the rise in serum $25 \mathrm{OHD} 3$ concentrations in relation to supplementation - a rise of $2 \mathrm{ng} / \mathrm{ml}$ for every 10,000 IU of vitamin D3, where vitD levels were inversely related to body weight (18). From this point of view, the dose of 1100 IU vitD administered by the present authors managed to cover the physiological body demand only, not the deficit as such.

Gronigen et al (21) developed a formula (dose (IU) $=40 \times(75-$ serum $250 \mathrm{HD} 3[\mathrm{nmol} / 1]) \times($ body weight $[\mathrm{kg}])$ for the calculation of vitD loading dose, based on empirical data from non-IBD subjects. For ordinary subjects with vitD insufficiency and approx. 80kg body weight, the minimum loading dose reaches 90000 IU D3, i.e. with fixed vitD doses used in our subjects and adequate compliance it may take at least 18 months to achieve normal serum levels.

The present study showed that a fixed vitD dosage of $1100 \mathrm{IU} /$ day regardless of vitD status was insufficient to overcome the deficit. Patient compliance with vitD supplementation was poor; however, it did not affect significantly the outcome with dosages used.

\section{References}

1. Bours PH, Wielders JP, Vermeijden JR, van de Wiel A. Seasonal variation of serum 25-hydroxyvitamin D levels in adult patients with inflammatory bowel disease. Osteoporos Int 2011; 22 (11): 2857-2867.

2. Yang L, Weaver V, Smith JP, Bingaman S, Hartman TJ, Cantorna MT. Therapeutic effect of vitamin D supplementation in a pilot study of Crohn's patients. Clin Transl Gastroenterol 2013; 4 (4): e33.

3. Jørgensen SP, Agnholt J, Glerup H et al. Clinical trial: vitamin D3 treatment in Crohn's disease a randomized double-blind placebo-controlled study. Aliment Pharmacol Ther 2010; 2: 377-383.
4. Kianicka B, Lata J, Novotny I, Dite P, Vanicek J. Single balloon enteroscopy for endoscopic retrograde cholangiography in patients with Roux-en-Y hepaticojejuno anastomosis. World J Gastroenterol 2013; 19 (44): 8047-8055.

5. Kianicka B, Dite P, Piskac P. Pitfalls of pancreatobiliary endoscopy after Billroth II gastrectomy. Hepatogastroenterology 2012; 1179: 17-21.

6. Kianicka B, Zak J, Bares M. The Use of Percutaneous Endoscopic Gastrostomy - Overview of Indications, Description of the Technique and Current Trends in Neurology. Ces Slov Neurol Neurochir 2012: 75 (2): 165-169.

7. Kianicka B, Dite P, Piskac P, Korbicka J, Vlcek P, Zak J. Endoscopic Approach in Diagnosis and Treatment of Biliary Complications after Laparoscopic Cholecystectomy. Hepato-gastroenterol 2011; 58 (106): 275-280.

8. Mouli VP, Ananthakrishnan AN. Review article: vitamin D and inflammatory bowel diseases. Aliment Pharmacol Ther 2014; 39: 125-136.

9. Miznerova E, Hlavaty T, Koller T et al. The prevalence and risk factors for osteoporosis in patients with inflammatory bowel disease. Bratisl Lek Listy 2013; 114 (8): 439-445.

10. Hintzpeter B, Mensink GB, Thierfelder W, Müller MJ, Scheidt-Nave C. Vitamin D status and health correlates among German adults. Eur J Clin Nutr 2008; 9: 1079-1089.

11. Dujsikova H, Dite P, Tomandl J, Sevcikova A, Precechtelova M. Occurrence of metabolic osteopathy in patients with chronic pancreatitis. Pancreatology 2008; 6: 583-586.

12. Farraye FA, Nimitphong H, Stucchi Aet al. Use of a novel vitamin $\mathrm{D}$ bioavailability test demonstrates that vitamin $\mathrm{D}$ absorption is decreased in patients with quiescent Crohn's disease. Inflamm Bowel Dis 2011; 17: 2116-2121.

13. Reich KM, Fedorak RN, Madsen K, Kroeker KI. Vitamin D improves inflammatory bowel disease outcomes: Basic science and clinical review. World J Gastroenterol 2014; 17: 4934-4947.

14. Ananthakrishnan AN, Cagan A, Gainer VS et al. Normalization of plasma 25-hydroxy vitamin $\mathrm{D}$ is associated with reduced risk of surgery in Crohn's disease. Inflamm Bowel Dis 2013; 19: 1921-1927.

15. an Assche G, Dignass A, Reinisch W et al. The second European evidence-based Consensus on the diagnosis and management of Crohn's disease: Special situations. J Crohn's Colitis 2010; 4: 63-101.

16. Hlavaty T, Krajcovicova A, Koller T, Toth J, Nevidanska M, Huorka M, Payer J. Higher vitamin D serum concentration increases health related quality of life in patients with inflammatory bowel diseases. World J Gastroenterol 2014; 42: 15787-15796.

17. Leichtmann GA, Bengoa JM, Bolt MJ, Sitrin MD. Intestinal absorption of cholecalciferol and 25-hydroxycholecalciferol in patients with both Crohn's disease and intestinal resection. Am J Clin Nutr 1991; 54 (3): 548-552.

18. Pappa HM, Mitchell PD, Jiang $\mathbf{H}$ et al. Treatment of vitamin D insufficiency in children and adolescents with inflammatory bowel disease: a randomized clinical trial comparing vitamin D2 three regimens. J Clin Endocrinol Metab 2012; 97: 2134-2142.

19. Lo CW, Paris PW, Clemens TL, Nolan J, Holick MF. Vitamin D absorption in healthy subjects and in patients with intestinal malabsorption. Am J Clin Nutr 1985; 42: 644-649.

20. Higgins PD, Rubin DT, Kaulback K, Schoenfield PS, Kane SV. Systematic review: impact of non-adherence to 5-aminosalicylic acid products on the frequency and cost of ulcerative colitis flares. Aliment Pharmacol Ther 2009; 29 (3): 247-257.

21. van Groningen L, Opdenoordt S, van Sorge A, Telting D, Giesen A, de Boer $\mathbf{H}$. Cholecalciferol loading dose guideline for vitamin D-deficient adults. Eur J Endocrinol 2010; 162: 805-811.

Received June 7, 2015. Accepted October 27, 2015. 